ESAIM: PROCEEDINGS, March 2013, Vol. 39, p. 1-6

M. Belhaq, P. Lafitte and T. Lelièvre Editors

\title{
GLOBAL ANALYSIS FOR A DELAY-DISTRIBUTED SIR EPIDEMIC MODEL
}

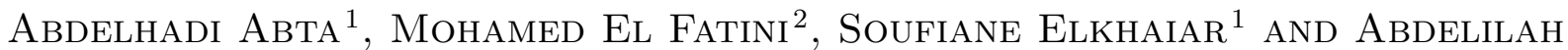 \\ $\mathrm{KADDAR}^{3}$
}

Résumé. En épidémiologie le retard joue un rôle très important dans la représentation de la période d'incubation. Dans ce travail, nous étudions un modèle SIR à retard distribué, avec une fonction d'incidence non linéaire. Pour analyser la stabilité globale du point d'équilibre sans maladie et du point d'équilibre endémique, nous utilisons la théorie de Lyapunov et le principe d'invariance de LaSalle.

\begin{abstract}
One of the most tools for describing the incubation period in the epidemiological dynamic models is the distributed delays. In this paper, we propose a delay-distributed SIR epidemic model with a nonlinear incidence rate. Using the Lyapunov theory and LaSalle's principle, we show the global asymptotic stability of the disease-free and the endemic equilibria. This analysis extends and performs existing results.
\end{abstract}

\section{SETTING THE PROBLEM}

The analysis of mathematical models describing the spread and control of infectious disease is one of major area biology. Transmission of a disease is a dynamical process driven by the interaction between susceptible and infective. The SIR infections disease models have been studied by many authors. The behavior of SIR models are greatly affected by the way in which transmission between infected and susceptible individuals. It divided the population being studied into three class labeled S, I and R, where S denotes the number of individuals who are susceptible to the disease, I denotes the number of infectious individuals and $\mathrm{R}$ denotes the number of individuals who had been infected and were removed from the possibility of being infected again or spreading infection. One fundamental parameter governs the spread of diseases, and is also related to the long term behaviors and the level of vaccination necessary for eradication. This parameter is called the "basic reproduction number", $R_{0} . R_{0}$ is defined by epidemiologists as "the average number of secondary cases caused by an infectious individual in a totally susceptible population". When $R_{0}>1$, the disease can enter a totally susceptible population and the number of cases will increase, whereas when $R_{0}<1$, the disease will always fail to spread. Therefore, in its simplest form $R_{0}$ tells us whether a population is at risk from a given disease. Nowadays, the results of many epidemiological research are presented in terms of basic reproduction number.

The epidemicity of disease is closely related to the stability of the solutions of the mathematical models. Given the global asymptotic stability conditions of those equilibria is of utmost biological relevance. Much attention has been paid to the analysis of the stability of the disease free equilibrium and the endemic equilibrium of the epidemic models. The fraction of papers that obtain global stability of these models is relatively few, especially the models with time delays $[2,12,15,19]$.

\footnotetext{
${ }^{1}$ Université Chouaib Doukkali, FS, BP.20, El Jadida, Morocco

2 Université Ibn Tofail, FS, LIRNE, BP. 133, Kénitra, Morocco.

${ }^{3}$ Université Mohammed V-Souissi, FSEJS, BP. 5285, Salé, Morocco
} 
In this paper, we consider an SIR epidemic model with distributed delay. An SIR model with distributed delay was studied in $[3,16,17]$. The distributed delay allows infectivity to be function of the duration since infection, up to some maximum duration. Here, we study an SIR model with nonlinear incidence. To analyze the stability of epidemic model, we use the Lyapunov's method.

The paper is organized as follows. In the next section, we present the model and derive the disease-free equilibrium and the endemic equilibrium. In the third section, we carry out a qualitative analysis of the model. Stability conditions for the disease-free equilibrium and the endemic equilibrium are derived. In the last section, we give some concluding remarks.

\section{The MAThematical MODEL}

In this work, we propose the following distributed delayed SIR epidemic model with a nonlinear incidence rate as follows :

$$
\left\{\begin{array}{l}
\frac{d S}{d t}=A-\mu S(t)-f(S(t), I(t)) \\
\frac{d I}{d t}=\int_{0}^{h} P(\tau) f(S(t-\tau), I(t-\tau)) d \tau-(\mu+\alpha+\gamma) I(t) \\
\frac{d R}{d t}=\gamma I(t)-\mu R(t)
\end{array}\right.
$$

where $\mathrm{P}$ is a nonnegative function such that $\int_{0}^{h} P(\tau) d \tau=1$.

The first two equations in system (1) do not depend on the third equation, and therefore this equation can be omitted without loss of generality. Hence, system (1) can be rewritten as

$$
\left\{\begin{array}{l}
\frac{d S}{d t}=A-\mu S(t)-f(S(t), I(t)) \\
\frac{d I}{d t}=\int_{0}^{h} P(\tau) f(S(t-\tau), I(t-\tau)) d \tau-(\mu+\alpha+\gamma) I(t)
\end{array}\right.
$$

The initial condition of delay differential equations (2) is given as :

$$
S(\theta)=\varphi_{1}(\theta), I(\theta)=\varphi_{2}(\theta), \theta \in[-h, 0]
$$

with $\varphi=\left(\varphi_{1}, \varphi_{2}\right) \in C^{+} \times C^{+}$, such that $\varphi_{i}(\theta) \geq 0(-\tau \leq \theta \leq 0, i=1,2)$. Here $C$ denotes the Banach space $C([-h, 0], \mathbb{R})$ of continuous functions mapping the interval $[-h, 0]$ into $\mathbb{R}$ equipped with the sup-norm. The nonnegative cone of $C$ is defined as $C^{+}=C\left([-h, 0], \mathbb{R}^{+}\right)$.

where $S$ is the number of susceptible individuals, $I$ is the number of infectious individuals, $R$ is the number of recovered individuals, $A$ is the recruitment rate of the population, $\mu$ is the natural death of the population, $\alpha$ is the death rate due to disease, $\gamma$ is the recovery rate of the infectious individuals, and $h$ is the incubation period.

In model (2) the formulation of the nonlinear incidence rate $f(S, I)$, i.e., the infection rate of susceptible individuals through their contacts with infectious, includes the three forms : The first one is the bilinear incidence rate $\beta S I$, where $\beta$ is the transmission rate [7]. The second one is the saturated incidence rate $\frac{\beta S I}{1+\alpha_{1} S+\alpha_{2} I}$, where $\alpha_{1}$ and $\alpha_{2}$ are the parameter that measure the inhibitory effect $[1,4,10,11,18,20]$. The third one is the standard incidence rate $\frac{\beta S I}{S+I+R}[6]$.

In [11], Kaddar et al. considered a local properties of a SIR model with constant delay and its corresponding SEIR model, and they observed that if $\mu \tau$ is close enough to 0 , then the delayed SIR and SEIR models generate identical local asymptotic stability. In [13], Korobeinikov established global stability for ordinary differential version of 2. In [9], Huang et al. have considered two epidemiological models with the constant time delays and nonlinear incidence rate of the form $f(S) g(I)$. In [8], Huang and Takeuchi established the global properties of 
epidemiological models with constant delays and nonlinear incidence rate $f(S, I)$. In [5], McCluskey established global stability for the following SIR epidemic model with distributed delays :

$$
\left\{\begin{array}{l}
\frac{d S}{d t}=A-\mu S(t)-\beta S(t) \int_{0}^{h} P(\tau) I(t-\tau) d \tau \\
\frac{d I}{d t}=\beta S(t) \int_{0}^{h} P(\tau) I(t-\tau) d \tau-(\mu+\alpha+\gamma) I(t)
\end{array}\right.
$$

In [6], Enatsu et al. established global asymptotic stability for the following SIR epidemic model with distributed delays :

$$
\left\{\begin{array}{l}
\frac{d S}{d t}=A-\mu S(t)-\int_{0}^{h} P(\tau) f(S(t), I(t-\tau)) d \tau, \\
\frac{d I}{d t}=\int_{0}^{h} P(\tau) f(S(t), I(t-\tau)) d \tau-(\mu+\alpha+\gamma) I(t) .
\end{array}\right.
$$

In this paper, by constricting an appropriate Lyapunov functionals, we estabilish the global asymptotic stability of a delayed SIR model (2).

We assume that $f: \mathbb{R}_{+}^{2} \longrightarrow \mathbb{R}_{+}^{2}$ is continuously differentiable in the interior of $\mathbb{R}_{+}^{2}$ satisfying $f(0, I)=f(S, 0)=0$ for $S, I \geq 0$ and the following hypotheses.

$\left(H_{1}\right): f(S, I)$ is a strictly monotone increasing function of $S \geq 0$, for any fixed $I>0$, and a monotone increasing function of $I \geq 0$, for any fixed $S \geq 0$,

$\left(H_{2}\right): \phi(S, I)=\frac{f(S, I)}{I}$ is bounded and monotone decreasing function of $I>0$, for any fixed $S \geq 0$, and $K(S)=\lim _{I \rightarrow 0^{+}} \phi(S, I)$ is continuous on $S \geq 0$.

We note that $K(S)>0$ holds for any $S>0$. System $(2)$ always has a disease-free equilibrium $E_{0}=\left(\frac{A}{\mu}, 0\right)$. On the other hand, under the hypotheses $(H 1)$ and $(H 2)$, if

$$
R_{0}=\frac{K\left(S_{0}\right)}{(\mu+\alpha+\gamma)}>1, S_{0}=\frac{A}{\mu}
$$

then system (2) also admits a unique positive equilibrium $E_{*}=\left(S^{*}, I^{*}\right)$, where $S, I$ satisfying the following equations (see Korobeinikov [13]).

$$
\begin{cases}A-\mu S^{*}-f\left(S^{*}, I^{*}\right) & =0 \\ f\left(S^{*}, I^{*}\right)-(\mu+\alpha+\gamma) I^{*} & =0\end{cases}
$$

Hereafter, we replace $\mu+\alpha+\gamma$ by $\delta$ and $\psi(t-\tau)$ by $\psi_{\tau}$ for any given function $\psi$.

Our main results are the following.

Théorème 0.1. Assume that the hypotheses $\left(H_{1}\right)$ and $\left(H_{2}\right)$ hold. Then the disease free equilibrium $E_{0}$ of the system (2) is the only equilibrium and globally asymptotically stable, if and only if $R_{0} \leq 1$.

Démonstration. We consider the following Lyapunov functional.

$$
\begin{aligned}
V(t)= & \int_{0}^{h} P(\tau) \int_{\frac{A}{\mu}}^{S_{\tau}}\left(1-\frac{K\left(\frac{A}{\mu}\right)}{K(u)}\right) d u d \tau+I(t) \\
& +\delta \int_{0}^{h} P(\tau) \int_{0}^{\tau} I_{u} d u d \tau .
\end{aligned}
$$


The time derivative of $V$ satisfies

$$
\begin{aligned}
\frac{d V(t)}{d t} & =\int_{0}^{h} P(\tau)\left(1-\frac{K\left(\frac{A}{\mu}\right)}{K\left(S_{\tau}\right)}\right) \dot{S}_{\tau} d \tau \\
& +\int_{0}^{h} P(\tau) f\left(S_{\tau}, I_{\tau}\right) d \tau-\delta I(t) \\
& +\delta \int_{0}^{h} P(\tau)\left[I(t)-I_{\tau}\right] d \tau \\
& =\int_{0}^{h} P(\tau)\left(1-\frac{K\left(\frac{A}{\mu}\right)}{K\left(S_{\tau}\right)}\right)\left(A-\mu S_{\tau}\right) d \tau \\
& +\delta \int_{0}^{h} P(\tau)\left(\frac{\phi\left(S_{\tau}, I_{\tau}\right)}{\delta} \frac{K\left(\frac{A}{\mu}\right)}{K\left(S_{\tau}\right)}-1\right) I_{\tau} d \tau .
\end{aligned}
$$

By the hypothesis $\left(H_{1}\right)$, we obtain that

$$
\mu\left(1-\frac{K\left(\frac{A}{\mu}\right)}{K\left(S_{\tau}\right)}\right)\left(\frac{A}{\mu}-S_{\tau}\right) \leq 0
$$

where strict equality holds if and only if $S(t)=S_{0}$. It follows from the hypothesis $(H 2)$ that

$$
\begin{aligned}
\frac{\phi\left(S_{\tau}, I_{\tau}\right)}{\delta} \frac{K\left(\frac{A}{\mu}\right)}{K\left(S_{\tau}\right)} & \leq \frac{K\left(S_{\tau}\right)}{\delta} \frac{K\left(\frac{A}{\mu}\right)}{K\left(S_{\tau}\right)} \\
& =\frac{K\left(\frac{A}{\mu}\right)}{\delta}=R_{0} .
\end{aligned}
$$

Then, $R_{0} \leq 1$ ensures that $\frac{d V(t)}{d t} \leq 0$ for all $t \leq 0$, where $\frac{d V(t)}{d t}=0$ holds if $S(t)=\frac{A}{\mu}$. It follows from system (2) that $E_{0}$ is the largest invariant set in $\left\{(S(t) ; I(t)) \in C \times C \mid \frac{d V(t)}{d t}=0\right\}$. From the Lyapunov-LaSalle's principle (Theorem 5.3, p. 30 in [14]), we obtain that $E_{0}$ is the only equilibrium of system (2) and globally asymptotically stable. This completes the proof.

\section{Global stability of the endemic equilibrium}

In this subsection, we give a proof of the global asymptotic stability of the endemic $E_{*}$ for $R_{0}>1$.

Théorème 1.1. Assume that the hypotheses $\left(H_{1}\right)$ and $\left(H_{2}\right)$ hold. If $R_{0}>1$, then the endemic equilibrium $E_{*}$ of system (2) is the only equilibrium and is globally asymptotically stable.

Démonstration. We define the following Lyapunov functional.

$$
\begin{aligned}
& V(t)=\int_{0}^{h} P(\tau) \int_{S^{*}}^{S_{\tau}}\left(1-\frac{f\left(S^{*}, I^{*}\right)}{f\left(u, I^{*}\right)}\right) d u d \tau \\
&+I(t)-I^{*}-I^{*} \ln \left(\frac{I(t)}{I^{*}}\right) \\
&+f\left(S^{*}, I^{*}\right) \int_{0}^{h} P(\tau) \int_{0}^{\tau} \frac{I_{u}}{I^{*}}-1-\ln \left(\frac{I_{u}}{I^{*}}\right) d u d \tau .
\end{aligned}
$$


Using the relation $A=\mu S^{*}+f\left(S^{*}, I^{*}\right)$ and $\delta I^{*}=f\left(S^{*}, I^{*}\right)$, the time derivative of the function $V(t)$ along the positive solution of system (2) becomes

$$
\begin{aligned}
\frac{d V(t)}{d t} & =\int_{0}^{h} P(\tau)\left(1-\frac{f\left(S^{*}, I^{*}\right)}{f\left(S_{\tau}, I^{*}\right)}\right) \dot{S}_{\tau} d \tau \\
& +\left(1-\frac{I^{*}}{I(t)}\right)\left(\int_{0}^{h} P(\tau) f\left(S_{\tau}, I_{\tau}\right) d \tau-\delta I(t)\right) \\
& +f\left(S^{*}, I^{*}\right) \int_{0}^{h} P(\tau)\left(\frac{I(t)}{I^{*}}-\frac{I_{\tau}}{I^{*}}+\ln \left(\frac{I_{\tau}}{I(t)}\right)\right) d \tau \\
& =\int_{0}^{h} P(\tau)\left(1-\frac{f\left(S^{*}, I^{*}\right)}{f\left(S_{\tau}, I^{*}\right)}\right)\left(\mu S^{*}-\mu S_{\tau}\right) d \tau \\
& +f\left(S^{*}, I^{*}\right) \int_{0}^{h} P(\tau)\left(1-\frac{f\left(S^{*}, I^{*}\right)}{f\left(S_{\tau}, I^{*}\right)}\right)\left(1-\frac{f\left(S_{\tau}, I_{\tau}\right.}{f\left(S^{*}, I^{*}\right)}\right) d \tau \\
& +f\left(S^{*}, I^{*}\right) \int_{0}^{h} P(\tau)\left(1-\frac{I^{*}}{I(t)}\right)\left(\frac{f\left(S_{\tau}, I_{\tau}\right)}{f\left(S^{*}, I^{*}\right)}-\frac{I(t)}{I^{*}}\right) d \tau \\
& +f\left(S^{*}, I^{*}\right) \int_{0}^{h} P(\tau)\left(\frac{I(t)}{I^{*}}-\frac{I_{\tau}}{I^{*}}+\ln \left(\frac{I_{\tau}}{I(t)}\right)\right) d \tau \\
& =\int_{0}^{h} P(\tau)\left(1-\frac{f\left(S^{*}, I^{*}\right)}{f\left(S_{\tau}, I^{*}\right)}\right)\left(\mu S^{*}-\mu S_{\tau}\right) d \tau \\
& +f\left(S^{*}, I^{*}\right) \int_{0}^{h} P(\tau)\left(2-\frac{f\left(S^{*}, I^{*}\right)}{f\left(S_{\tau}, I^{*}\right)}+\frac{f\left(S_{\tau}, I_{\tau}\right)}{f\left(S_{\tau}, I^{*}\right)}\right. \\
& \left.-\frac{I^{*} f\left(S_{\tau}, I_{\tau}\right)}{I(t) f\left(S^{*}, I^{*}\right)}-\frac{I_{\tau}}{I^{*}}+\ln \left(\frac{I_{\tau}}{I(t)}\right)\right) d \tau .
\end{aligned}
$$

Here by using

$$
\ln \left(\frac{I_{\tau}}{I(t)}\right)=\quad \ln \left(\frac{f\left(S^{*}, I^{*}\right)}{f\left(S_{\tau}, I^{*}\right)}\right)+\ln \left(\frac{I^{*} f\left(S_{\tau}, I_{\tau}\right)}{I(t) f\left(S^{*}, I^{*}\right)}\right)+\ln \left(\frac{I_{\tau} f\left(S_{\tau}, I^{*}\right)}{I^{*} f\left(S_{\tau}, I_{\tau}\right)}\right)
$$

a straightforward calculations give

$$
\begin{aligned}
\frac{d V(t)}{d t} & =\int_{0}^{h} P(\tau)\left(1-\frac{f\left(S^{*}, I^{*}\right)}{f\left(S_{\tau}, I^{*}\right)}\right)\left(\mu S^{*}-\mu S_{\tau}\right) d \tau \\
& +f\left(S^{*}, I^{*}\right) \int_{0}^{h} P(\tau)\left(1-\frac{f\left(S^{*}, I^{*}\right)}{f\left(S_{\tau}, I^{*}\right)}+\ln \left(\frac{f\left(S^{*}, I^{*}\right)}{f\left(S_{\tau}, I^{*}\right)}\right)\right) d \tau \\
& +f\left(S^{*}, I^{*}\right) \int_{0}^{h} P(\tau)\left(1-\frac{I^{*} f\left(S_{\tau}, I_{\tau}\right)}{I(t) f\left(S^{*}, I^{*}\right)}+\ln \left(\frac{I^{*} f\left(S_{\tau}, I_{\tau}\right)}{I(t) f\left(S^{*}, I^{*}\right)}\right)\right) d \tau \\
& +f\left(S^{*}, I^{*}\right) \int_{0}^{h} P(\tau)\left(1-\frac{I_{\tau} f\left(S_{\tau}, I^{*}\right)}{I^{*} f\left(S(t-\tau), I_{\tau}\right)}+\ln \left(\frac{I_{\tau} f\left(S_{\tau}, I^{*}\right)}{I^{*} f\left(S_{\tau}, I_{\tau}\right)}\right)\right) d \tau \\
& +f\left(S^{*}, I^{*}\right) \int_{0}^{h} P(\tau)\left(\frac{I_{\tau}}{I^{*}}-\frac{f\left(S_{\tau}, I_{\tau}\right)}{f\left(S_{\tau}, I^{*}\right)}\right)\left(\frac{f\left(S_{\tau}, I^{*}\right)}{f\left(S_{\tau}, I_{\tau}\right)}-1\right) d \tau .
\end{aligned}
$$

From hypothesis $\left(H_{1}\right)$, we have

$$
\left(1-\frac{f\left(S^{*}, I^{*}\right)}{f\left(S_{\tau}, I^{*}\right)}\right)\left(\mu S^{*}-\mu S_{\tau}\right) \leq 0
$$

and from hypotheses $\left(H_{1}\right)$ and $\left(H_{2}\right)$, we have

$$
\begin{aligned}
& \left(\frac{I_{\tau}}{I^{*}}-\frac{f\left(S_{\tau}, I_{\tau}\right)}{f\left(S_{\tau}, I^{*}\right)}\right)\left(\frac{f\left(S_{\tau}, I^{*}\right)}{f\left(S_{\tau}, I_{\tau}\right)}-1\right)=\frac{I_{\tau}}{I^{*} \phi\left(S_{\tau}, I^{*}\right) f\left(S_{\tau}, I_{\tau}\right)} \times \\
& \left(\phi\left(S_{\tau}, I^{*}\right)-\phi\left(S_{\tau}, I_{\tau}\right)\right)\left(f\left(S_{\tau}, I^{*}\right)-f\left(S_{\tau}, I_{\tau}\right)\right) \leq 0 .
\end{aligned}
$$


since the function $g(x)=1-x+\ln (x)$ is always non-positive for any $x>0$, and $g(x)=0$ if and only if $x=1$. We conclude that, $\frac{d V(t)}{d t}$ is non-positive. Hence, the functional $V$ satisfies all the conditions of Theorem 5.3 of Kuang [14]. This proves that $E_{*}$ is globally asymptotically stable.

\section{Conclusion}

In epidemiological research literatures, a latent or incubation period can be medelled by incorporating it as a delay effect (SIR models with constant or distributed time delay), or by introducing an exposed class (SEIR models).

In this paper we considered the global stability of an SIR epidemiological model with nonlinear incidence rate and distributed time delays (system (1)). We established the global asymptotic stability by using an appropriate Lyapunov functional techniques. For $R_{0} \leq 1$, we showed the global stability of disease-free equilibrium in Theorem 1 and for $R_{0}>1$, we obtained the sufficient conditions of the endemic equilibrium in Theorem 2 .

\section{REFERENCES}

[1] R. M. Anderson and R. M. May; Regulation and stability of host-parasite population interactions : I. Regulatory processes, The Journal of Animal Ecology, Vol. 47, no. 1, pp. 219-267, 1978.

[2] E. Beretta, Y. Takeuch; Global stability of an SIR epidemic model with time delays, Journal of Mathematical Biology, 33(3) (1995), 250-260.

[3] E. Beretta, T. Hara, W. Ma, Y. Takeuchi; Global asymptotic stability of an SIR epidemic model with distributed time delay, Nonlinar Anal. 47 (2001), 4107-4115.

[4] V. Capasso, G. Serio ; A generalization of Kermack-Mckendrick deterministic epidemic model, Math. Biosci. Vol. 42, pp. 41-61, 1978.

[5] C. Connell McCluskey; Complete global stability for an SIR epidemic model with delay-distributed or discrete, Nonlinear Analysis : Real World Applications 11 (2010) 55-59.

[6] Y. Enatsu, Y. Nakata and Y. Muroya Global stability of SIR epidemic models with a wide class of nonlinear incidence and distributed delays, Discrete and Continuous Dynamical Systems Series B Volume 15, Number 1, (2011) 61-74.

[7] M. Gabriela, M. Gomes, L. J. White, G. F. Medley; The reinfection threshold, Journal of Theoretical Biology, Vol. 236, pp. $111-113,2005$.

[8] G. Huang, Y. Takeuchi Global Analysis on Delay Epidemiological Dynamic Models with Nonlinear Incidence, Journal of Mathematical Biology, (2010) 72 :1192-1207.

[9] G. Huang, Y. Takeuchi, W. Ma Global Stability for delay SIR and SEIR epidemic models Delay Epidemiological Dynamic Models with nonlinear incidence rate, Journal of Bull. Math. Biol., (2011) 63(1), p. 125-139.

[10] Z. Jiang, J. Wei ; Stability and bifurcation analysis in a delayed SIR model, Chaos, Solitons and Fractals, Vol. 35, pp. 609-619, 2008.

[11] A. Kaddar, A. Abta, H. Talibi Alaoui A comparison of delayed SIR and SEIR epidemic models, Nonlinear Analysis : Modelling and Control, 2011, Vol. 16, No. 2, 181-190.

[12] A. Kaddar, A. Abta, H. Talibi Alaoui Global stability for delay SIR and SEIR epidemic models with saturated incidence rates, Electronic Journal of Diferential Equations, Vol. 2012 (2012), No. 23, pp. 1-13.

[13] A. Korobeinikov Lyapunov Functions and Global Stability for SIR and SIRS Epidemiological Models with Non-linear Transmission, Bulletin of Mathematical biology (2006) 30:615-626.

[14] Y.Kuang Delay Differentiel Equations With Applications in Population Dynamics, Academic Press, INC., New York 1993.

[15] W. B. Ma, M. Song and Y. Takeuchi; Global Stability of an SIR Epidemic Model with Time Delay, Applied Mathematics Letters, 17 (2004), 1141-1145.

[16] Y. Takeuchi, W. B. Ma, E. Beretta; Global asymptotic properties of a delay SIR epidemic model with finite incubation times, Nonlinear Anal. 42 (2000), 931-947.

[17] W. B. Ma, Y. Takeuchi, T. Hara, E. Beretta; Permanence of an SIR epidemic model with distributed time delays, Tohoku Math. 54 (2002), 581-591.

[18] R. Xu, Z. Ma; Stability of a delayed SIRS epidemic model with a nonlinear incidence rate, Chaos, Solitons and Fractals, Vol. 41, Iss. 5, pp. 2319-2325, 2009.

[19] S. L. Yuan and Z. E. Ma; Global stability and hopf bifurcation of an SIR epidemic model with time delays, Journal of System science and Complexity, 14 (2001), 327-336.

[20] F. Zhang, Z. Z. Li, F. Zhang; Global stability of an SIR epidemic model with constant infectious period, Applied Mathematics and Computation, Vol. 199, pp. 285-291, 2008. 